\title{
Contribution to safety analyses of DEMO HCPB using AINA code
}

\author{
Eduard Baeza ${ }^{\mathrm{a}}$, Alfredo de Blas ${ }^{\mathrm{a}}$, Albert Riego ${ }^{\mathrm{a}}$, Marco Fabbri ${ }^{\mathrm{a}}$ \\ ${ }^{a}$ Fusion Energy Engineering Laboratory, Technical University of Catalonia (UPC) Barcelona-Tech, Barcelona, Spain
}

\begin{abstract}
The motivation of the current work, framed under the safety EUROfusion activities to develop DEMO, is to present the conclusions drawn from our contribution to the safety studies of the HCPB DEMO carried out by the team tasked with AINA code development. During 2016 and 2017 a new AINA version was built in order to evaluate plasma evolution and in-vessel components strains inside the European DEMO designs. As a result, AINA is able to foresee several accident scenarios as plasma disruptions or structural meltings due to LOPCs (Loss Of Plasma Control) and in-vessel melt either of FW, blanket structure and/or divertor modules because of thermal stresses due to LOCAs. After due analysis, it has concluded that it would be desirable to carry out a design review focused on ensuring a suitable operating temperature range with a bigger safety margin for all the materials which make up the $\mathrm{HCPB} \mathrm{BB}$, as well as the need to guarantee a quick detection and actuation by means of a proper system, depending on the affected equipment, when the most demanding transients take place which may drive the reactor to melting scenarios and very energetic plasma disruptions. These events include an increase of fueling above $50 \%$, a permanent improvement in the confinement time and a punctual impurity increase above $300 \%$. Other perturbations has been studied which provide information on non-dangerous cases, impossible situations or melting processes.
\end{abstract}

Keywords: DEMO, HCPB, AINA, safety analysis, LOPC, LOCA.

\section{Introduction}

The safety and environmental goals of a fusion power plant design are to protect workers from radiation, electromagnetic fields, chemical and other hazards; the public from radioactive and toxic materials and the environment from pollutants and waste. A conclusion that can be drawn from the historical safety analyses developed for tokamaks is that some of the major risks involve incidents in the vacuum vessel and during the last ten years AINA has become a great tool in order to evaluate plasma evolution and these in-vessel components strains [1-6]. The Blanket is a safety relevant component since its failure could impact the operation of other components, most notably the vacuum vessel. Moreover, the Blanket is credited for some interlock functions such as neutron shielding to the magnets that may affect the dose to the workers during maintenance operations, and hence its design could impact the overall safety of the machine. Thus, this contribution to the HCPB safety studies is faced to figure out if the in vessel components fulfill the safety functions and to foresee the possible consequences if these functions are not satisfied.

Firstly, it is important to highlight that the tool used to perform this study, AINA 4.0, has been properly validated $[7,8]$ and the modeled Blanket is the HCPB-2015 v3 [9].

\section{Steady State}

The steady state scenario used to develop the safety study was presented in the previous AINA team's paper [7] and based on the reference scenario DEMO1 [10]. It is necessary to remember that for the DEMO1 scenario certain functional temperature limits for the HCPB BB design are slightly exceeded in the worst poloidal region (EUROFER: $563^{\circ} \mathrm{C}$, Beryllium: $677^{\circ} \mathrm{C}$ and LiSiO4: 956 $\left.{ }^{\circ} \mathrm{C}\right)$. These values are slightly different from previous paper ones due to and improvement in the thermal blanket model. Accordingly, it would be advisable to undertake a design review focused on ensuring a suitable operating temperature for materials which make up the blanket [7].

\section{Transients}

\subsection{Types of accidents}

Common objective of the FFMEAs done for the various DEMO systems was, at first, to provide a complete list of potential accident initiating events (IEs). Among those, the Selection of reference accident scenarios for the DEMO plant document [11] has identified 21 PIEs (Postulated Initiating Events) as the most representative for the deterministic assessments to be performed in the first phase of the DEMO design activities both to check the compliance with safety limits and to give rationales for the selection of the reference DEMO reactor model. The vast majority of these 21 PIEs could induce the following load or accident scenarios which AINA is able to simulate:

- Plasma disruption or structural material melting due to a LOPC (Loss Of Plasma Control): disruptions represent the highest risk for DEMO integrity and it is assumed that they are only caused by instabilities induced by failures in the systems operating to confine, diagnose and feed the plasma or by accidental events leading to the entrance of undesired elements. By means of AINA, it is possible to identify those failures which lead to the maximum wall damage due to the electromagnetic and thermal load. Likewise, some failures cannot lead the plasma to a disruption event, however this perturbation can 
affect the plasma physics and damage structural materials. The perturbations that AINA can simulate are failures in the external power system and in the fuel system, changes in the confinement time and an undesired entrance of impurities.

- In-vessel melt either of FW, blanket structure and/or divertor modules because of thermal stresses due to a LOCA: AINA is able to replicate this phenomena by means of the variation of the coolant mass flow rate through the cooling sections while the reactor stills working.

\subsection{Failure in the external power supply system}

An unexpected and instantaneous auxiliary heating cut-off while the HCPB DEMO reactor is operating on DEMO1 steady state causes a fast decrease of ion and electron temperatures and a confinement time rise. All of this converges at a new steady state which produces a higher fusion power $\left(\mathrm{P}_{\text {fus }}\right)$ of $2420 \mathrm{MW}$. So, apparently the ignition point has been achieved, however the increase of neutronic heat flux (NWL) through the blanket induces the overtaking of the functional temperature limits wider than in the DEMO1 state in less than $10 \mathrm{~s}\left(\mathrm{LiSiO}_{4}: 1031\right.$ ${ }^{\circ} \mathrm{C}$, Beryllium: $700{ }^{\circ} \mathrm{C}$ and EUROFER: $565^{\circ} \mathrm{C}$ ). For this reason it is necessary to conclude that it is really important to detect rapidly an external power cut-off in order to proceed with a fast plasma shutdown (FPSS) during the first seconds for the purpose of preventing, specifically, $\mathrm{LiSiO}_{4}$ and Beryllium melting.

An analogous and scaled sequence to the previous one takes place when an unexpected and sudden decrease of auxiliary heating takes place. In short, any auxiliary heating reduction must be detected instantly in order to proceed with a FPSS to avoid the BB material collapse.

If the reactor suffers an unexpected and sudden increase of auxiliary heating there are two situations. When the increase is up to a multiplication factor of 6.3 the reactor is driven to an initial and abrupt fusion power increment although it finally decreases rapidly achieving a new state which produces less fusion power than DEMO1 and where the functional temperature limits of the BB materials are closer to the accepted ones. When the increase by a multiplication factor is larger than 6.3 the plasma terminates disruptively due to the beta limit infringement. The higher multiplication factor is, the faster plasma collapse is. At the time of the disruption, the plasma thermal energy (U) (estimated by Paknezhad expression (1) [12] where $\mathrm{Z}$ indicates the plasma species, $\mathrm{n}_{\mathrm{z}}$ is the specie density, $\mathrm{T}_{\mathrm{z}}$ is the temperature density and $\mathrm{V}$ is the plasma volume) is 1.5 GJ. Consequently the EUROFER limit of $550{ }^{\circ} \mathrm{C}$ would be significantly exceeded as well as the rest of the temperature limits.

$$
U=\frac{3}{2} \sum_{Z} n_{Z} T_{Z} V
$$

Nevertheless and in spite of these consequences, it is highly unlikely to achieve auxiliary heating as high as those which can lead to this final kind of accident due to the installed external power would not exceed $150 \mathrm{MW}$.
Table 1. Auxiliary heating perturbation summary.

\begin{tabular}{|c|c|c|c|}
\hline $\begin{array}{l}\text { Mult. } \\
\text { factor }\end{array}$ & $\begin{array}{l}\text { Plasma } \\
\text { termination }\end{array}$ & Mitigation & Consequences \\
\hline 0 & Stabilized & FPSS & $\begin{array}{c}P_{\text {fus }} \text { increase/ } \\
\text { melting }\end{array}$ \\
\hline 0.5 & Stabilized & FPSS & $\begin{array}{l}P_{\text {fus }} \text { increase/ } \\
\text { melting }\end{array}$ \\
\hline 2 & Stabilized & - & $P_{\text {fus }}$ decrease \\
\hline 5 & Stabilized & - & $P_{\text {fus }}$ decrease \\
\hline 6.4 & $\begin{array}{l}\text { Beta limit at } \\
88.5 \mathrm{~s}\end{array}$ & FPSS & $\begin{array}{l}\text { Melting by } \\
\text { excessive U }\end{array}$ \\
\hline 9 & $\begin{array}{l}\text { Beta limit at } \\
2 \mathrm{~s}\end{array}$ & FPSS & $\begin{array}{l}\text { Melting by } \\
\text { excessive U }\end{array}$ \\
\hline
\end{tabular}

\subsection{Failure in the fuel source system}

In case fueling is stopped, it is possible to observe how $P_{\text {fus }}$ quickly diminish as well as densities, except the impurities percentages due to physical sputtering driven by the increase in ion and electron temperature. All of this leads to a reduction in $\mathrm{BB}$ temperatures reaching acceptable values far below the limits. Unfortunately, a transition L mode is detected after $22.9 \mathrm{~s}$ and, finally, plasma collapses at $25.5 \mathrm{~s}$ by an overshoot beta limit disruption. Despite this, at the time of the collapse, the $U$ achieves values of up to 0.5 GJ. This released energy could be mitigated by a disruption mitigation system and, thereby, the BB material would be safeguarded.

A fueling reduction leads to a fusion power decrease, specifically half of fueling provides just over half the initial $\mathrm{P}_{\text {fus }}$ (from $2037 \mathrm{MW}$ to $1141 \mathrm{MW}$ ). The confinement time gets stabilized during the first seconds and material temperatures are greatly reduced even almost below the functional temperature limits. In conclusion, beyond this failure does not cause a dangerous scenario, the new steady state obtained could be a better candidate for DEMO operation from the point of view of temperature limits no exceedance and despite the generation of lower $\mathrm{P}_{\text {fus }}$ and, consequently, less gain.

If an unexpected and sudden increase of fueling rate up to $25 \%$ a new steady state scenario is achieved as in the previous case. However this one is not positive and several risks are derived from this kind of perturbation since $\mathrm{P}_{\text {fus }}$ expands as well as confinement time and densities. All of this leads to a scenario where functional temperature limits are widely exceeded in less than $50 \mathrm{~s}$ and the melting is unmitigated $\left(\mathrm{LiSiO}_{4}: 1212{ }^{\circ} \mathrm{C}\right.$, Beryllium: $764{ }^{\circ} \mathrm{C}$ and EUROFER: $572.5^{\circ} \mathrm{C}$ for a rate up of $25 \%$ ). Hence, it is essential to be able to guarantee a quick detection and actuation (FPSS) in order to stop the excessive warming.

When an unexpected and sudden increase of fueling rate is above $25 \%, \mathrm{P}_{\text {fus }}$, densities and confinement time are continuously growing until a Greenwald limit disruption takes place with a high $U(>1 \mathrm{GJ})$. The higher fueling rate multiplication factor is, the earlier collapse happens. In short, it is necessary to detect an increase of fueling rapidly and activate a fast plasma shutdown (FPSS) since all these perturbations cause structural damage. 
Table 2. Fueling rate perturbation summary.

\begin{tabular}{|c|c|c|c|}
\hline $\begin{array}{l}\text { Mult. } \\
\text { factor }\end{array}$ & $\begin{array}{l}\text { Plasma } \\
\text { termination }\end{array}$ & Mitigation & Consequences \\
\hline 0 & $\begin{array}{l}\text { Beta limit at } \\
25.5 \mathrm{~s}\end{array}$ & $\begin{array}{l}\text { Disruption } \\
\text { mitigation }\end{array}$ & - \\
\hline 0.5 & Stabilized & - & $\begin{array}{l}\text { New and } \\
\text { better SS }\end{array}$ \\
\hline 1.25 & Stabilized & FPSS & $\begin{array}{l}\mathrm{P}_{\text {fus }} \text { increase/ } \\
\text { melting }\end{array}$ \\
\hline 2 & $\begin{array}{l}\text { Greenwald } \\
\text { limit at } 74.7 \mathrm{~s}\end{array}$ & FPSS & $\begin{array}{l}\text { Melting by } \\
\text { excessive U }\end{array}$ \\
\hline 6 & $\begin{array}{l}\text { Greenwald } \\
\text { limit at } 1.7 \mathrm{~s} \\
\end{array}$ & FPSS & $\begin{array}{l}\text { Melting by } \\
\text { excessive U }\end{array}$ \\
\hline
\end{tabular}

\subsection{Variations in the confinement time}

Historically, AINA has been used in safety analyses to scan the influence of an increase in confinement time [3, $6,13]$ owing to the $\mathrm{H}$ mode discovery occurred on 4th February 1982. Following this path, at first, the behavior of plasma parameters in case of a permanent increase of confinement time will be simulated and discussed.

Confinement time improvement produces a fast increase of temperatures as well as densities, which drive to an overall raise of $\mathrm{P}_{\text {fus }}$ until plasma terminates disruptively due to the beta limit infringement. The $U$ discharged during the disruption cause structural damage and melting in any case, hence, it is necessary to detect instantaneously an unexpected variation in confinement time with the aim of conducing the reactor to a FPSS.

Table 3. Confinement time permanent improvement summary.

\begin{tabular}{llccc}
\hline $\begin{array}{l}\text { Mult. } \\
\text { factor }\end{array}$ & $\begin{array}{l}\text { Termination } \\
\text { time (s) }\end{array}$ & $\begin{array}{l}\mathrm{U} \\
(\mathrm{GJ})\end{array}$ & $\begin{array}{l}\mathrm{Max} \mathrm{P}_{\text {fus }} \\
(\mathrm{MW})\end{array}$ & $\begin{array}{l}\mathrm{Max} . \mathrm{LiSiO}_{4} \\
\mathrm{~T}\left({ }^{\circ} \mathrm{C}\right)\end{array}$ \\
\hline 1.5 & 31.7 & 2.4 & 4708 & 1475 \\
2 & 5.5 & 2.4 & 4905 & 1432 \\
3 & 3.7 & 2.4 & 5332 & 1408 \\
5 & 3 & 2.4 & 5565 & 1388 \\
\hline
\end{tabular}

On the other hand a punctual confinement time variation may happen due to an unexpected behavior during the operation time. The impact of these events is highly dependent on the duration of the confinement time variation and it does not depend so much on the variation factor suffered. Therefore, when the confinement time rises abruptly but decreases after $0.1 \mathrm{~s}$, the effects are not severe since both a multiplication factor of 3 and 10 excites the plasma but the initial steady state is recovered in less than $20 \mathrm{~s}$. During this transition an instantaneous $P_{\text {fus }}$ peak is reached due to a fast increase of ion and electron temperatures, as well as the BB materials temperatures rise slightly but they are stabilized rapidly. In case the duration of the confinement time variation is longer, the steady state is recovered whilst the perturbation disappears before the corresponding termination time tracked down in the previous section with the exception of the perturbation lasts more than a boundary value $(\sim 3 \mathrm{~s})$ where the plasma may collapse due to the Greenwald limit. In these cases and in spite of the recovery, the longer the duration of the perturbation is the grater maximum temperatures are achieved and it is highly probable that the reactor suffers a melting situation. In view of these circumstances, it is necessary to detect an unexpected variation in confinement time.

\subsection{Entrance of an undesired quantity of impurities}

When the reactor is operating, a flux of particles enter the plasma as impurities due to the erosion phenomena which is inherent to the nature of plasma wall interaction. It can be cause of several undesirable effects as a Plasma Facing Components damage or beneficial effects as shutting down the fusion due to an increase of Bremsstrahlung and line radiation, becoming a passive safety mechanism, for instance, if a LOCA takes place. For these reasons the consequences of an undesired quantity of impurities must be studied.

A punctual increase of the impurities presence (Xe and W) up to $300 \%$ produces a sudden rise of confinement time, $\mathrm{P}_{\text {fus, }}$ power losses (Bremsstrahlung and line radiation); a decrease of temperatures and, after a few seconds, the achievement of dangerous melting temperatures on the BB materials. A recovery of the initial steady state takes place after 20 seconds; however, the detection of this kind of perturbation and a FPSS must be fast since, as noted, the material temperatures rise rapidly.

A punctual increase of the impurities presence (Xe and W) equal to $300 \%$ differs from the previous case, the plasma equilibrium is not recovered and plasma skips instantaneous to the $\mathrm{L}$ mode where $\mathrm{P}_{\text {fus }}$ grows until a peak of $4365 \mathrm{MW}$. Consequently, the temperature in the BB rises rapidly $\left(\mathrm{LiSiO}_{4}\right.$ : $1395{ }^{\circ} \mathrm{C}$, Beryllium: $771{ }^{\circ} \mathrm{C}$, EUROFER: $572{ }^{\circ} \mathrm{C}$ and Tungsten: $534.8{ }^{\circ} \mathrm{C}$ ). Densities are continuously growing until a Greenwald limit disruption takes place at $12 \mathrm{~s}$ releasing a $\mathrm{U}$ of $1.8 \mathrm{GJ}$ which can damage the reactor structure. So, it is necessary to detect this event rapidly and lead the reactor to a FPSS.

Finally, if an increase of the Tungsten impurity production takes place, $\mathrm{P}_{\text {fus }}$, densities and confinement time are continuously and slowly growing and depending on the new impurity production rate the plasma may reach a new steady state or it might collapse by means of the Greenwald limit. Nevertheless, any alternative is not instantaneous, so the incident detection must be previous to the endpoint in order to prevent a melting scenario.

\subsection{LOCAs}

Firstly, it is important to remember that AINA's LOCAs are based on the variation of the coolant mass flow rate through the cooling sections while the reactor stills working and not after a reactor shut down.

From AINA outcomes, it concludes that this kind of failure inside the cooling system does not affect the internal plasma conditions but, undoubtedly, even a slight loss of the mass flow (about 30\%), leads the reactor to an overall melting scenario rapidly since the material temperatures increase drastically during the first seconds after the cooling channel rupture. Specifically, the most demanded area is the $\mathrm{LiSiO}_{4}$ layer due to its lower thermal conductivity and a big distance from the cooling channel 
lines, consequently, the effect of the convention is less effective than in the other layers. For this reason, it is indispensable the installation of a quick response system capable of detecting a cooling anomaly rapidly and activating a proper mitigation action such as a FPSS.

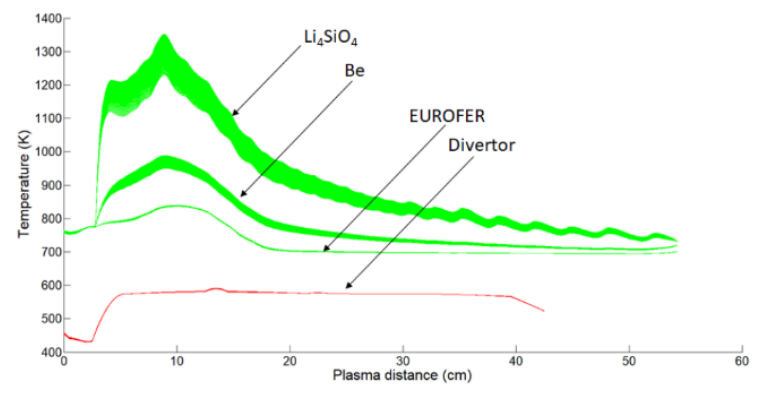

Fig. 1. T Evolution of the BB if a 30\% BB LOCA occurs.

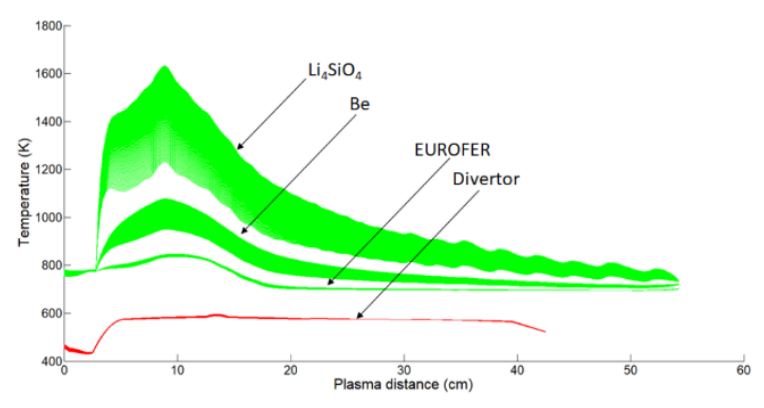

Fig. 2. T Evolution of the BB if a $60 \%$ BB LOCA occurs.

\section{Conclusions}

After due analysis, it has concluded that it would be desirable to carry out a possible design review to ensure suitable operating temperatures for all the materials which make up the HCPB BB, as well as the need to guarantee a quick detection and actuation by means of a proper system, depending on the affected equipment, when the most demanding transients take place which may drive the reactor to suffer a melting scenario and a very energetic plasma disruption at the same time. These events include an increase of fueling injection above $50 \%$, a permanent improvement in the confinement time and a punctual impurity increase above $300 \%$. Other perturbations has been studied, which some of them only provide information on non-dangerous cases as a decrease of the fueling injection, impossible situations from a technical point of view as an unexpected and sudden increase of external power above $630 \%$ or melting processes as LOCAs or a decrease of the external power injection.

\section{Future Work}

On the basis of this HCPB study, future tasks will be focused on the development of the DCLL, HCLL and WCLL contributions to safety analyses using AINA 4.0.

This work has been carried out within the framework of the EUROfusion Consortium and has received funding from the Euratom research and training programme 20142018 under grant agreement No 633053. The views and opinions expressed herein do not necessarily reflect those of the European Commission.

\section{References}

[1] J. Dies, M. Dapena, M. Ramon, J. Garcia, J.C. Rivas, A. Calvo, S. Reyes, AINA safety code, a review of loss of plasma control transients in ITER: sudden increase in fueling rate, sudden increase of auxiliary heating, Fusion Science and Technology. 56 (2009) 31-37.

[2] J.C. Rivas, J. Dies, Upgrading of plasma wall interaction model for tokamak transient modeling code AINA 2.0, used in safety studies of ITER plasma instability events, Fusion Science and Technology. 60 (2011) 825-829.

[3] J.C. Rivas, J. Dies, Safety studies: Review of loss of plasma control transients in ITER with AINA 3.0 code, Fusion Engineering and Design. 88 (2013) 2709-2713. http://dx.doi.org/10.1016/j.fusengdes.2013.02.134

[4] J.C. Rivas, J. Dies, ITER safety studies: The effect of two simultaneous perturbations during a loss of plasma control transient, Fusion Engineering and Design. 89 (2014) 2043-2047.

http://dx.doi.org/10.1016/j.fusengdes.2014.04.010.

[5] J.C. Rivas, J. Dies, X. Fajarnés, Revisiting the analysis of passive plasma shutdown during an ex-vessel loss of coolant accident in ITER blanket, Fusion Engineering and Design. 98-99 (2015) 2206-2209.

http://dx.doi.org/10.1016/j.fusengdes.2015.06.143

[6] J.C. Rivas, M. Nakamura, Y. Someya, K. Hoshino, N. Asakura, H. Takase, Y. Miyoshi, H. Utoh, K. Tobita, J. Dies, A. de Blas, A. Riego, M. Fabbri, Safety studies of plasma-wall events with AINA code for Japanese DEMO, Fusion Engineering and Design. (2015).

https://dx.doi.org/10.1016/j.fusengdes.2015.10.037

[7] E. Baeza, A. de Blas, A. Riego, M. Fabbri, Development of the safety code AINA for the European DEMO designs, Fusion Engineering and Design. (2018). doi:10.1016/J.FUSENGDES.2018.04.074.

[8] M. Fabbri, A. de Blas, A. Riego, I. Zamora, E. Baeza, Methodology for the improvement of the AINA Code wall-model applied to DEMO WCPB blanket, Fusion Engineering and Design, ISSN 0920-3796, (2017). http://dx.doi.org/10.1016/j.fusengdes.2017.05.027

[9] F.A. Hernández, Q. Kang, B. Kiss, P. Norajitra, G. Nádasi, P. Pereslavtsev, C. Zeile, HCPB Design Report 2015. Internal_Deliverable_BB-1.2.1-T002, (EUROfusion document, Working Package of Safety and Environment).

[10] R. Wenninger, DEMO1 Reference Design - 2015 April ("EU DEMO1 2015"), 2MDKFH. (EUROfusion document, Working Package of Safety and Environment).

[11] T. Pinna, Selection of reference accident scenarios for the DEMO plant. 2MA6TU_v1_0, (EUROfusion document, Working Package of Safety and Environment).

[12] A. Paknezhad, A.S. Elahi, M. Ghoranneviss, Plasma Thermal Energy Measurement based on the Plasma Diamagnetic Effect in the IR-T1 Tokamak, 3 (2013) 145148. doi:10.5923/j.jnpp.20130305.04.

[13] J.C. Rivas, Development of AINA code for the study of loss of plasma control events in ITER and DEMO, and contribution to the systems study of DEMO, PhD thesis, Universitat Politècnica de Catalunya, 2016. 